\title{
Variable selection procedures before partial least squares regression enhance the accuracy of milk fatty acid composition predicted by mid-infrared spectroscopy
}

\author{
P. Gottardo, ${ }^{*}$ M. Penasa, ${ }^{* 1}$ N. Lopez-Villalobos, $\dagger$ and M. De Marchi ${ }^{*}$ \\ *Department of Agronomy, Food, Natural Resources, Animals and Environment, University of Padova, Viale dell'Università 16, \\ 35020 Legnaro (PD), Italy \\ †Institute of Veterinary, Animal and Biomedical Sciences, Massey University, Private Bag 11 222, Palmerston North, 4442, New Zealand
}

\begin{abstract}
Mid-infrared spectroscopy is a high-throughput technique that allows the prediction of milk quality traits on a large-scale. The accuracy of prediction achievable using partial least squares (PLS) regression is usually high for fatty acids (FA) that are more abundant in milk, whereas it decreases for FA that are present in low concentrations. Two variable selection methods, uninformative variable elimination or a genetic algorithm combined with PLS regression, were used in the present study to investigate their effect on the accuracy of prediction equations for milk FA profile expressed either as a concentration on total identified FA or a concentration in milk. For FA expressed on total identified FA, the coefficient of determination of cross-validation from PLS alone was low (0.25) for the prediction of polyunsaturated FA and medium (0.70) for saturated FA. The coefficient of determination increased to 0.54 and 0.95 for polyunsaturated and saturated FA, respectively, when FA were expressed on a milk basis and using PLS alone. Both algorithms before PLS regression improved the accuracy of prediction for FA, especially for FA that are usually difficult to predict; for example, the improvement with respect to the PLS regression ranged from 9 to $80 \%$. In general, FA were better predicted when their concentrations were expressed on a milk basis. These results might favor the use of prediction equations in the dairy industry for genetic purposes and payment system.
\end{abstract}

Key words: genetic algorithm, milk fatty acid, midinfrared spectroscopy, variable selection

Received January 3, 2016.

Accepted July 3, 2016.

${ }^{1}$ Corresponding author: mauro.penasa@unipd.it

\section{INTRODUCTION}

Infrared technologies are fast, cheap, and largely used to determine a large number of milk characteristics (De Marchi et al., 2014; Visentin et al., 2015; McDermott et al., 2016a,b). Methods such as partial least squares (PLS) and principal component regression are useful to extract relevant information from the spectra and potentially build accurate and robust models using the whole spectrum (Geladi and Kowalski, 1986; Thomas and Haaland, 1990). These methods are considered almost insensitive to noise and, therefore, it is a common belief that variable selection is not necessary for models construction (Haaland and Thomas, 1988); however, in the last decade some researchers have suggested that an efficient variable selection is useful and sometimes necessary to obtain suitable prediction models for both routine and research purposes (Martens and Naes, 1989; Helland, 2001; Chun and Keleş, 2010). In fact, PLS regression is a projection-based method because it ignores the direction in the variable space where noisy and irrelevant variables are present; however, when the number of predictor variables is much greater than the number of observations, this property of the PLS estimator ceases to exist. Faber et al. (1995) studied the error propagation in principal component analysis and reported that the bias of the model is greatly influenced by the number of predictor variables and by the measurement error; if several uninformative variables are present (i.e., a model with a lot of noise) the final PLS estimators will be biased.

Prediction of concentration of individual fatty acids (FA) in cow milk using mid-infrared spectroscopy (MIRS) applying PLS is robust and precise for the major FA groups and for some individual FA, but the quality of the prediction decreases for FA that are present in low concentrations (Soyeurt et al., 2006, 2011; De Marchi et al., 2011). The application of a variable selection procedure before PLS regression could lead to a better and less complex prediction model (Mehmood 
et al., 2012). The present study compared 2 different methods of variables selection, namely the uninformative variable elimination (UVE) procedure proposed by Centner et al. (1996) and a genetic algorithm (GA). The UVE procedure has been already tested with satisfactory results on titratable acidity, calcium content, and detailed protein composition of bovine milk (Gottardo et al., 2015; Niero et al., 2016); GA has been mainly used on traits predicted by near infrared spectroscopy, but also reviewed and developed for MIRS by Leardi and González (1998) and tested on milk FA composition by Ferrand et al. (2011). The main goal of the present study was to investigate which of the 2 methods is the best to select variables and thus to be practically implemented under field conditions.

\section{MATERIALS AND METHODS}

\section{Sample Collection and MIRS Acquisition}

Individual milk samples of 63 Holstein-Friesian, 24 Brown Swiss, and 25 Jersey cows from parity 1 to 7 and from 7 to 408 DIM were collected in 4 herds between February and March 2015 during the morning milking. Preservative (Bronopol, 2-bromo-2-nitropropan-1,3diol; Grunenthal Prodotti \& Farmaceutici Formenti, Milan, Italy) was immediately added to milk, which was then transferred at $4^{\circ} \mathrm{C}$ to the laboratory of the South Tirol Dairy Association (Bolzano, Italy) and analyzed for milk chemical composition using a MilkoScan FT6000 (Foss Electric A/S, Hillerød, Denmark). For each sample, the absorbance spectrum contained 1,060 infrared data points over the spectral range from 900 to $5,000 \mathrm{~cm}^{-1}$. An aliquot of each sample was transferred to the laboratory of the Department of Agronomy, Food, Natural Resources, Animals and Environment of the University of Padova (Legnaro, Italy) for FA analysis.

\section{Milk FA Analysis}

Milk lipids were determined with accelerated solvent extraction method using Dionex ASE 350 system (Thermo Scientific, Dreieich, Germany) with petroleum ether in isopropanol (2:1) as solvent. Methyl esterification of FA was carried out according to Palmquist and Jenkins (2003) with a basic or acid reaction. Fatty acid separation and quantification were performed by a Agilent 7820A GC System equipped with an automatic sampler G4567A (Agilent Technologies, Santa Clara, $\mathrm{CA}$ ) and flame ionization detector. The column used was a Supelco Omegawax capillary column $(30 \mathrm{~m}$ in length, $0.25-\mathrm{mm}$ inner diameter and a film thickness of $0.25 \mu \mathrm{m}$; Supelco, Bellefonte, PA). Temperatures of injector and flame ionization detector were set at $250^{\circ} \mathrm{C}$. Oven temperature was initially $50^{\circ} \mathrm{C}$ for $2 \mathrm{~min}$, increased at $4^{\circ} \mathrm{C} / \mathrm{min}$ to $220^{\circ} \mathrm{C}$, and held for $18 \mathrm{~min}$. Hydrogen was the carrier gas and its flow was set at $1 \mathrm{~mL} / \mathrm{min}$ with average speed of $21 \mathrm{~cm} / \mathrm{s}$. Fatty acid standard Supelco FAME mixC4-C24 \#18919-1AMP (Sigma-Aldrich, Castle Hill, Australia) was analyzed before GC analysis for FA identification. Determination of FA values was obtained using GC ChemStation software (Agilent Technologies, Santa Clara, CA) and were expressed both as total identified FA and on a milk basis. Individual FA were C4:0, C6:0, C8:0, C12:0, C14:0, C16:0, C16:1n-7, C18:0, and C18:1n-9, and groups of FA were SFA, UFA, MUFA, and PUFA.

\section{Statistical Analysis}

Spectral Information. Spectral data were transformed to absorbance using the $\log _{10}$ of transmittance value and the 1,060 wavenumbers were reduced to 480 through the elimination of 2 spectra regions (1,601 to 1,717 and 3,052 to $5,011 \mathrm{~cm}^{-1}$ ) known to be related to water absorption and, thus, characterized by high noise (Hewavitharana and van Brakel, 1997). Prediction equations were derived based on PLS regression using the ChemometricsWithR package (Wehrens, 2011) and the possible presence of outliers was checked using the robust Mahalanobis distance procedure. Following this procedure, no outliers were detected in the data set. Due to the quite low spectral number of observations, we decided to perform a leave-one-out cross-validation instead of an external validation. Wavenumbers to be included in as dependent variables of the PLS regression were selected based on 2 methods, UVE and GA. Both UVE and GA were run using R (64 bit) statistical software (R Core Team, 2015). The UVE procedure was performed using a homemade script whereas GA using the rgba.bin function implemented in the genalg package of $\mathrm{R}$.

UVE Procedure. The UVE procedure was proposed by Centner et al. (1996) and involves the addition of artificial noise variables to the original wavelength matrix of predictors to create a filter. All the original variables having less stability than the noisy artificial variables are eliminated. The procedure is repeated until a stop criterion is reached. The optimal number of principal components must be set for each trait before starting the PLS procedure and we decided to use 10 of them for each trait. This choice was taken to avoid possible overfitting issues due to the low number of samples in the present study. The UVE procedure consists of 6 steps. 
1. Generation of a random noise matrix with dimensions $N \times p$, where $N$ is the number of observations and $p$ is the number of predictors (wavenumbers) in the data set. Random entries must be generated from a uniform distribution in the interval 0.0 to 1.0 and multiplied by a very small constant $\left(10^{-10}\right.$ in our case).

2. Combination of the original wavenumber matrix with the pseudorandom numbers matrix, which leads to a new matrix called $\mathbf{Z}$, with dimension $N \times 2 p$.

3. Application of a PLS regression on the $\mathbf{Z}$ matrix using a leave-one-out cross-validation with the optimal number of principal components determined as above. The root mean square error of cross-validation $\left(\mathbf{R M S E}_{\mathbf{C V}}\right)$ was registered in a separate vector. It was defined as

$$
\mathrm{RMSE}_{\mathrm{CV}}=\sqrt{\frac{\sum_{i=1}^{n}\left(\hat{y}_{i}-y_{i}\right)^{2}}{N-k-1}},
$$

where $y_{i}$ is the actual concentration of a FA, $\hat{y}_{i}$ is the predicted concentration of the FA, $N$ is the number of samples, and $k$ is the number of principal components retained in the model.

4. Use of the leave-one-out coefficients obtained through jack-knifing to compute a stability criterion $\left(\mathbf{c}_{\mathbf{j}}\right)$ for each variable as

$$
\mathrm{c}_{\mathrm{j}}=\frac{\operatorname{mean}\left(\hat{\beta}_{j}\right)}{\mathrm{SD}\left(\hat{\beta}_{j}\right)}, \text { for } j=1, \ldots, 2 p,
$$

where mean $\left(\hat{\beta}_{j}\right)$ and $\operatorname{SD}\left(\hat{\beta}_{j}\right)$ are the average and standard deviation of the vector of $n$ coefficients obtained by leave-one-out jack-knifing validation.

5. Setting the filter using the absolute higheststability criterion among random noisy variables as the threshold value. If the absolute higheststability criterion of a random variable is greater than the one of an original variable, then the original variable is uninformative and discarded from the model.

6. Performing a new PLS with the remaining variables and check the model performances using the RMSE $\mathrm{CV}_{\mathrm{CV}}$. If the new $\mathrm{RMSE}_{\mathrm{CV}}$ is larger than $\mathrm{RMSE}_{\mathrm{CV}}$ obtained from point 3, the algorithm stops, otherwise the procedure restarts from point 1 , but with $\mathrm{A}-1$ principal components.
$\boldsymbol{G A}$. Genetic algorithm is a heuristic approach that combines Mendelian genetics and Darwinian evolution to find a nearby optimal solution through the simulation of these 2 principles. There is not an ideal GA, as the success of the algorithm is strictly dependent on how well it has been adapted to the specific problem to be solved (Leardi, 2003). The basic idea of GA is quite simple; variables that produce models with better-fitting statistics have higher probability to survive and to be reused on another generation of solutions. Variables (wavenumbers in our case) take the name of genes and the whole-spectra chromosome. After a fixed number of generations or when the fitting statistics remain constant for a predecided consecutive number of generations, the algorithm will stop. The main risks of GA are over-fitting and random correlation among variables. Some criteria have been proposed to avoid these problems. According to Leardi (2003), the original number of variables must not exceed 200 and the ratio of observations to variables must not be greater than 5 . To meet these requirements we reduced the data to 160 dependent variables (wavenumbers) by using the mean of 3 contiguous wavenumbers and GA was performed on this new data set. The GA was carried out according to the following steps.

1. Generation of an initial population of genes expressed by bits where 1 represents the selected variable and 0 the not-selected variable. The selection of the initial population was performed using a pseudorandom cycle that selected an average of 5 genes per chromosome. A maximum of 30 genes per chromosome were selected.

2. Generation of 30 chromosomes as solutions through a pseudorandom step.

3. Fitting a PLS model to each solution and running a full leave-one-out cross-validation using a fixed number of principal components (10), as in the UVE procedure.

4. Retention of the best solutions (i.e., solutions with the lowest $\mathrm{RMSE}_{\mathrm{CV}}$ ), defined as above. We retained the $20 \%$ best solutions. These solutions will survive until the next generation.

5. Mutation was applied with a probability of $1 \%$ per gene (one unselected variable becomes selected and vice versa).

6. Creation of a new generation: a new pool of solutions including the best $20 \%$ of the previous generation.

7. This new set of variables serves as input for point 3.

8. Steps 3 to 5 were repeated 100 times (100 generations). 
To avoid a suboptimal variable selection, the procedure was repeated 30 times. The variables were selected according to the percentage of their selection during each of the 30 runs and a threshold of $80 \%$ was chosen for this task.

Fitting Statistics. The goodness of fit of the equations in the cross-validation data sets were checked according to $\mathrm{RMSE}_{\mathrm{CV}}$, as described above, and the coefficient of determination in leave-one-out cross-validation $\left(\mathrm{R}_{\mathrm{CV}}^{2}\right)$, calculated as

$$
\mathrm{R}_{\mathrm{CV}}^{2}=\frac{\sum_{i=1}^{N}\left(\hat{y}_{i}-\bar{y}\right)^{2}}{\sum_{i=1}^{N}\left(y_{i}-\bar{y}\right)^{2}},
$$

where $\bar{y}$ is the mean of the actual values. A 2-sample Kolmogorov-Smirnov test was performed to check if $\mathrm{R}_{\mathrm{CV}}^{2}$ obtained from the statistical approaches were significantly different $(P<0.05)$. This means that 3 comparisons were performed within each trait (PLS vs. UVE + PLS, PLS vs. GA + PLS, and UVE + PLS vs. GA + PLS).

In addition, the practical utility of prediction models was investigated through the ratio performance deviation (RPD):

$$
\mathrm{RPD}=\mathrm{SD} / \mathrm{RMSE}_{\mathrm{CV}},
$$

where SD is the standard deviation of reference values of the trait. Values of RPD greater than 2 are consid- ered adequate for analytical purposes (Sinnaeve et al., 1994).

\section{RESULTS AND DISCUSSION}

\section{FA Composition}

Means and SD of FA expressed as grams per $100 \mathrm{~g}$ of total identified FA and grams per $100 \mathrm{~mL}$ of milk are presented in Tables 1 and 2, respectively. The composition of both FA groups and individual FA was consistent with findings reported by several authors (De Marchi et al., 2011; Ferrand et al., 2011; Soyeurt et al., 2011; Comino et al., 2015). Saturated FA were the most abundant followed by MUFA and PUFA. Among individual FA, C16:0 and C18:1n-9 were the most represented and averaged 29.78 and $18.78 \mathrm{~g} / 100 \mathrm{~g}$ of total identified FA and 0.99 and $0.60 \mathrm{~g} / 100 \mathrm{~mL}$ of milk, respectively. The coefficient of variation for FA groups and individual FA expressed on total identified FA ranged from 0.05 (SFA) to 0.23 (C16:1n-7), and for FA groups and individual FA expressed on a milk basis it ranged from 0.25 (C8:0) to 0.40 (C12:0 and $\mathrm{C} 16: 1 \mathrm{n}-7)$. Overall, the traits exhibited an appreciable variation, which facilitated the development of prediction models.

\section{Selected Variables}

Both UVE and GA procedures selected wavenumbers that corresponded to the absorption areas closely associated with FA: the first region between 2,823 and 3,016

Table 1. Cross-validation fitting statistics ${ }^{1}$ obtained by partial least squares (PLS) regression only, uninformative variable elimination (UVE)

\begin{tabular}{|c|c|c|c|c|c|c|c|c|c|c|c|c|}
\hline \multirow[b]{2}{*}{ Trait } & \multirow[b]{2}{*}{ Mean (SD) } & \multicolumn{3}{|c|}{ PLS } & \multicolumn{4}{|c|}{ UVE + PLS } & \multicolumn{4}{|c|}{$\mathrm{GA}+\mathrm{PLS}$} \\
\hline & & No. of PC & $\mathrm{R}_{\mathrm{CV}}^{2}$ & $\mathrm{RMSE}_{\mathrm{CV}}$ & $\mathrm{w}$ & $\mathrm{R}_{\mathrm{CV}}^{2}$ & $\mathrm{RMSE}_{\mathrm{CV}}$ & $\mathrm{RPD}$ & $\mathrm{w}$ & $\mathrm{R}_{\mathrm{CV}}^{2}$ & $\mathrm{RMSE}_{\mathrm{CV}}$ & $\mathrm{RPD}$ \\
\hline \multicolumn{13}{|c|}{ Group of FA } \\
\hline SFA & $67.53(3.46)$ & 10 & 0.70 & 1.897 & 105 & 0.78 & 1.551 & 2.23 & 35 & 0.82 & 1.390 & 2.49 \\
\hline UFA & $32.47(3.42)$ & 10 & 0.65 & 2.251 & 184 & 0.71 & 1.878 & 1.82 & 44 & 0.75 & 1.746 & 1.95 \\
\hline MUFA & $27.12(3.42)$ & 10 & 0.61 & 2.644 & 150 & 0.70 & 1.863 & 1.83 & 41 & 0.75 & 1.700 & 2.01 \\
\hline PUFA & $5.35(1.08)$ & 10 & 0.25 & 1.103 & 82 & 0.50 & 0.720 & 1.46 & 32 & 0.54 & 0.690 & 1.57 \\
\hline C6:0 & $1.76(0.24)$ & 10 & 0.70 & 0.164 & 160 & 0.80 & 0.120 & 2.00 & 38 & 0.82 & 0.102 & 2.40 \\
\hline $\mathrm{C} 8: 0$ & $1.20(0.18)$ & 10 & 0.57 & 0.214 & 135 & 0.71 & 0.100 & 1.80 & 46 & 0.74 & 0.092 & 2.00 \\
\hline C12:0 & $3.14(0.58)$ & 10 & 0.70 & 0.323 & 95 & 0.80 & 0.262 & 2.23 & 35 & 0.84 & 0.240 & 2.42 \\
\hline C14:0 & $10.04(1.18)$ & 10 & 0.52 & 0.956 & 174 & 0.69 & 0.651 & 1.82 & 45 & 0.75 & 0.576 & 2.03 \\
\hline C16:0 & $29.78(2.72)$ & 10 & 0.55 & 1.622 & 145 & 0.68 & 1.552 & 1.75 & 42 & 0.73 & 1.403 & 1.94 \\
\hline C18:0 & $10.99(2.20)$ & 10 & 0.40 & 1.581 & 150 & 0.55 & 1.414 & 1.56 & 48 & 0.60 & 1.390 & 1.58 \\
\hline C16:1n-7 & $1.43(0.33)$ & 10 & 0.38 & 0.452 & 144 & 0.52 & 0.232 & 1.43 & 35 & 0.54 & 0.224 & 1.50 \\
\hline
\end{tabular}
+ PLS regression, or genetic algorithm (GA) + PLS regression for milk fatty acid (FA) composition expressed in g/100 g of total identified FA

${ }^{1}$ No. of $\mathrm{PC}=$ optimal number of principal components; $\mathrm{R}_{\mathrm{CV}}^{2}=$ coefficient of determination in leave-one-out cross-validation; $\mathrm{RMSE} \mathrm{CV}_{\mathrm{CV}}=$ root mean square error in cross-validation; $\mathrm{w}=$ number of variables (wavenumbers) selected after UVE and GA procedures; RPD $=$ ratio performance deviation, calculated by dividing the $\mathrm{SD}$ of reference values to the $\mathrm{RMSE}_{\mathrm{CV}}$ of the trait. 
Table 2. Cross-validation fitting statistics ${ }^{1}$ obtained by partial least squares (PLS) regression only, uninformative variable elimination (UVE) + PLS regression, or genetic algorithm (GA) + PLS regression for milk fatty acid (FA) composition expressed in g/100 mL of milk

\begin{tabular}{|c|c|c|c|c|c|c|c|c|c|c|c|c|}
\hline \multirow[b]{2}{*}{ Trait } & \multirow[b]{2}{*}{ Mean (SD) } & \multicolumn{3}{|c|}{ PLS } & \multicolumn{4}{|c|}{$\mathrm{UVE}+\mathrm{PLS}$} & \multicolumn{4}{|c|}{$\mathrm{GA}+\mathrm{PLS}$} \\
\hline & & No. of PC & $\mathrm{R}_{\mathrm{CV}}^{2}$ & $\mathrm{RMSE}_{\mathrm{CV}}$ & $\mathrm{w}$ & $\mathrm{R}_{\mathrm{CV}}^{2}$ & $\mathrm{RMSE}_{\mathrm{CV}}$ & $\mathrm{RPD}$ & $\mathrm{w}$ & $\mathrm{R}_{\mathrm{CV}}^{2}$ & $\mathrm{RMSE}_{\mathrm{CV}}$ & RPD \\
\hline SFA & $2.24(0.61)$ & 10 & 0.95 & 0.122 & 350 & 0.99 & 0.091 & 6.78 & 50 & 0.99 & 0.091 & 6.78 \\
\hline UFA & $1.05(0.30)$ & 10 & 0.80 & 0.182 & 138 & 0.90 & 0.100 & 3.00 & 48 & 0.95 & 0.072 & 4.28 \\
\hline MUFA & $0.90(0.27)$ & 10 & 0.80 & 0.140 & 190 & 0.90 & 0.090 & 3.00 & 41 & 0.95 & 0.064 & 4.50 \\
\hline PUFA & $0.15(0.05)$ & 10 & 0.54 & 0.054 & 90 & 0.66 & 0.032 & 1.70 & 30 & 0.71 & 0.020 & 2.50 \\
\hline C6:0 & $0.06(0.02)$ & 10 & 0.92 & 0.005 & 149 & 0.93 & 0.005 & 4.00 & 43 & 0.94 & 0.005 & 4.00 \\
\hline C8:0 & $0.04(0.01)$ & 10 & 0.93 & 0.003 & 125 & 0.93 & 0.003 & 3.33 & 39 & 0.94 & 0.002 & 4.18 \\
\hline C12:0 & $0.10(0.04)$ & 10 & 0.82 & 0.022 & 68 & 0.90 & 0.010 & 4.00 & 29 & 0.93 & 0.008 & 4.65 \\
\hline C14:0 & $0.33(0.09)$ & 10 & 0.89 & 0.033 & 40 & 0.92 & 0.030 & 3.61 & 25 & 0.93 & 0.020 & 3.89 \\
\hline C16:0 & $0.99(0.30)$ & 10 & 0.90 & 0.090 & 300 & 0.91 & 0.088 & 3.36 & 45 & 0.92 & 0.084 & 3.64 \\
\hline C18:0 & $0.36(0.13)$ & 10 & 0.70 & 0.090 & 252 & 0.79 & 0.062 & 2.17 & 37 & 0.80 & 0.064 & 2.26 \\
\hline $\mathrm{C} 16: 1 \mathrm{n}-7$ & $0.05(0.02)$ & 10 & 0.71 & 0.043 & 100 & 0.79 & 0.012 & 2.25 & 40 & 0.82 & 0.007 & 2.30 \\
\hline
\end{tabular}

${ }^{1}$ No. of $\mathrm{PC}=$ optimal number of principal components; $\mathrm{R}_{\mathrm{CV}}^{2}=$ coefficient of determination in leave-one-out cross-validation; $\mathrm{RMSE} \mathrm{EV}_{\mathrm{CV}}=$ root mean square error in cross-validation; $\mathrm{w}=$ number of variables (wavenumbers) selected after UVE and GA procedures; RPD $=$ ratio performance deviation, calculated by dividing the $\mathrm{SD}$ of reference values to the $\mathrm{RMSE}_{\mathrm{CV}}$ of the trait.

$\mathrm{cm}^{-1}$ and the second region between 1,736 and 1,805 $\mathrm{cm}^{-1}$. The absorption area between 1,736 and 1,805 $\mathrm{cm}^{-1}$ is of particular importance because it corresponds to the zone of absorption of carbonyl groups (Coates, 2000). On average, the UVE algorithm selected more wavenumbers (160) than GA (114). Also, UVE retained more wavenumbers on FA expressed on a milk basis (172) than as grams per $100 \mathrm{~g}$ of total identified FA (140).

\section{Fitting Statistics}

Calibration equations of FA composition expressed as grams per $100 \mathrm{~g}$ of total identified FA (Table 1) were less accurate; that is, they exhibited lower $\mathrm{R}_{\mathrm{CV}}^{2}$ than equations of FA composition expressed as grams per $100 \mathrm{~mL}$ of milk (Table 2). This result is mainly related to a different dispersion of values for FA concentration in milk and milk fat; that is, 2 milk samples could have the same fat profile but different fat percentage in milk (Soyeurt et al., 2006; De Marchi et al., 2014).

Prediction models for FA composition obtained by UVE + PLS and GA + PLS regressions exhibited better-fitting statistics than models developed using PLS regression only, and GA + PLS led to better results than UVE + PLS (Tables 1 and 2). For traits expressed on total identified $\mathrm{FA}$, the $\mathrm{R}_{\mathrm{CV}}^{2}$ from PLS, $\mathrm{UVE}+\mathrm{PLS}$, and GA + PLS regression ranged from 0.25 (PUFA) to 0.70 (SFA, C6:0 and C12:0), 0.50 (PUFA) to 0.80 (C6:0 and C12:0), and 0.54 (PUFA and C16:1n-7) to 0.84 (C4:0 and C12:0), respectively (Table 1). When expressed on a milk basis, the lowest $R_{C V}^{2}$ were estimated for PUFA (0.54, 0.66, and 0.71 for PLS, UVE + PLS, and GA + PLS regression, respectively) and the greatest were obtained for SFA $(0.95,0.99$, and 0.99, for PLS, UVE + PLS, and GA + PLS regression, respectively; Table 2).

Compared with PLS regression, the UVE + PLS approach improved $\mathrm{R}_{\mathrm{CV}}^{2}$ of prediction models of $\mathrm{SFA}$, UFA, MUFA, and PUFA expressed as grams per $100 \mathrm{~g}$ of total identified FA by $11,9,15$, and $67 \%$, respectively $(P<0.05$; Figure 1$)$; these percentages increased to $17,15,23$, and $80 \%$, respectively, when the GA + PLS approach was used $(P<0.05)$. When expressed on a milk basis, UFA and MUFA exhibited percentage variations that were similar to those calculated on the same traits expressed on total identified FA, whereas the percentage variation of PUFA was much less pronounced $(P<0.05)$. Finally, the accuracy of SFA expressed on a milk basis improved marginally and not significantly compared with PLS regression only (Figure 2). Compared with UVE + PLS, GA + PLS regression improved the $\mathrm{R}_{\mathrm{CV}}^{2}$ of prediction models for $\mathrm{FA}$ groups between 5 (SFA) and 8\% (MUFA) when expressed as grams per $100 \mathrm{~g}$ of total identified FA $(P<$ 0.05 ), and between 0 (SFA) and 8\% (PUFA; $P<0.05)$ when expressed on a milk basis. The $\mathrm{R}_{\mathrm{CV}}^{2}$ for individual FA expressed as grams per $100 \mathrm{~g}$ of total identified FA increased between 9 (C4:0) and 38\% (C18:0) when UVE + PLS instead of PLS regression only was used, and the increment was always significant $(P<0.05)$. These values ranged from 17 (C6:0) to 50\% (C18:0) when GA + PLS was applied $(P<0.05$; Figure 1$)$. The accuracy of FA expressed on a milk basis was less influ- 
enced by the statistical approach compared with FA on total identified FA (Figure 2). Overall, it appeared that the 2 methods performed better on traits that were poorly predicted by PLS regression only.

Fitting statistics for FA expressed on a milk basis were comparable to those reported by Ferrand et al. (2011) after a GA optimization, except for PUFA, which were much better predicted in the present study $\left(\mathrm{R}_{\mathrm{CV}}^{2}=0.71\right)$ than in Ferrand et al. $(2011)\left(\mathrm{R}_{\mathrm{CV}}^{2}=0.38\right)$.
To our knowledge, no other studies dealt with selection algorithms for variable selection before PLS analysis for assessing bovine milk FA composition. Several authors (e.g., Soyeurt et al., 2006, 2011; De Marchi et al., 2011; Lopez-Villalobos et al., 2014) used PLS regression on MIRS spectra collected to predict FA profile of bovine milk expressed on a fat or milk basis. However, the use of UVE + PLS or GA + PLS coupled with leave-oneout cross-validation in the present study makes any
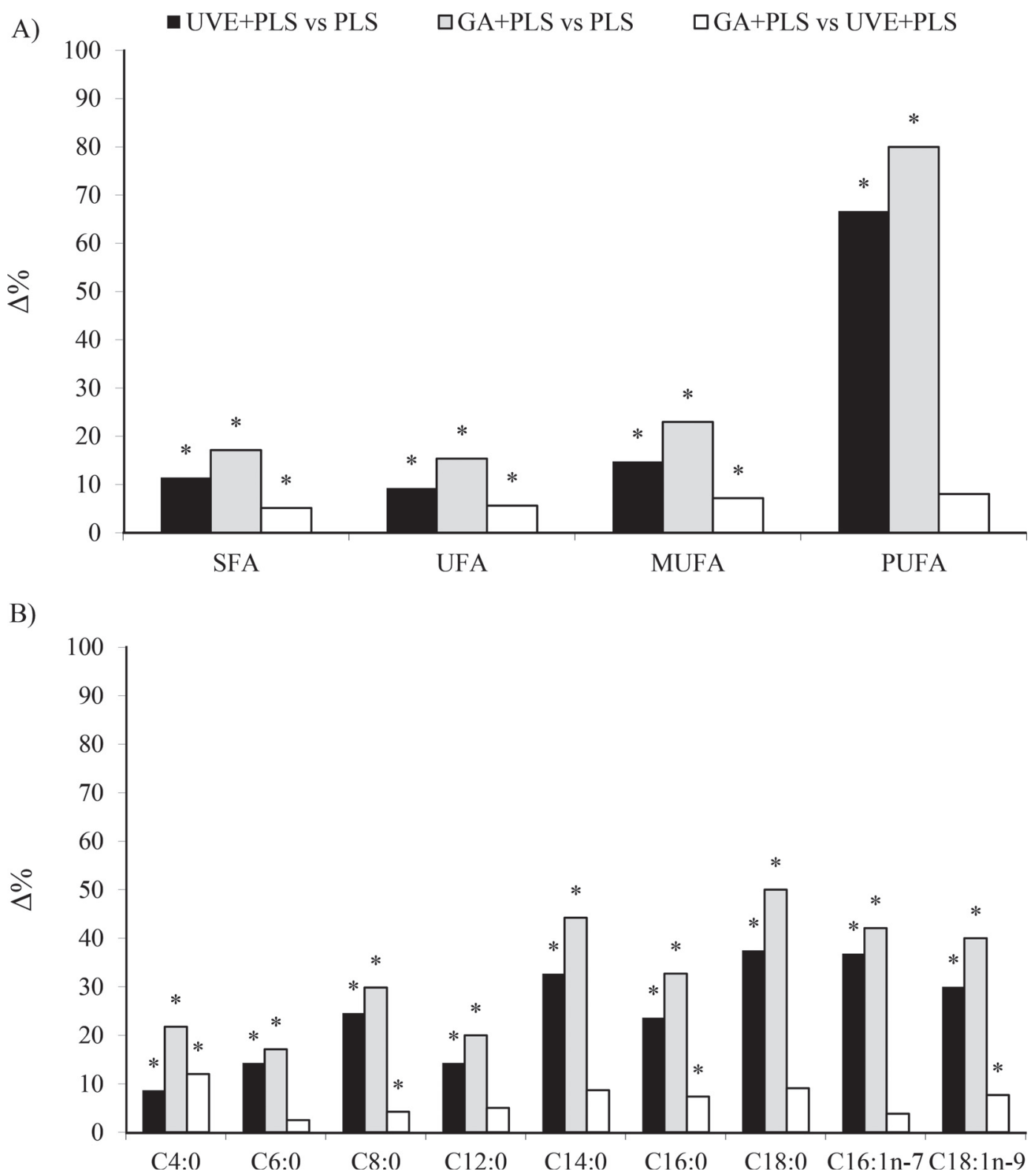

Figure 1. Percentage variation $(\Delta \%)$ of coefficient of determination in leave-one-out cross-validation $\left(\mathrm{R}_{\mathrm{CV}}^{2}\right)$ of prediction models for $(\mathrm{A})$ milk fatty acids (FA) groups and (B) individual milk FA (expressed in $\mathrm{g} / 100 \mathrm{~g}$ of total identified FA) obtained by comparing uninformative variable elimination (UVE) + partial least squares (PLS) regression versus PLS regression, genetic algorithm (GA) + PLS regression versus PLS regression, and GA + PLS regression versus UVE + PLS regression. The asterisk $\left(^{*}\right)$ above the bars indicates that percentage variations of coefficient of determination in leave-one-out cross-validation were significantly different at $P<0.05$. 


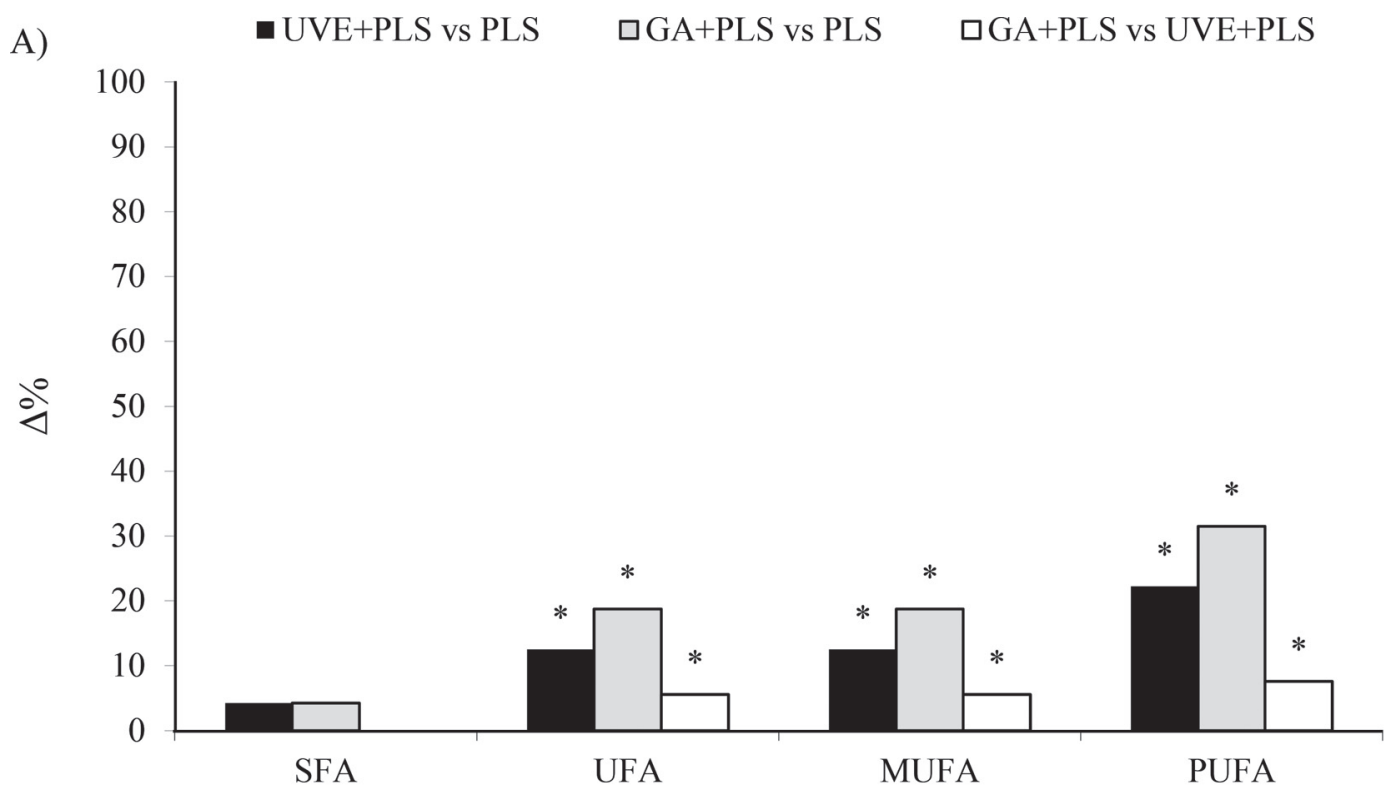

B)

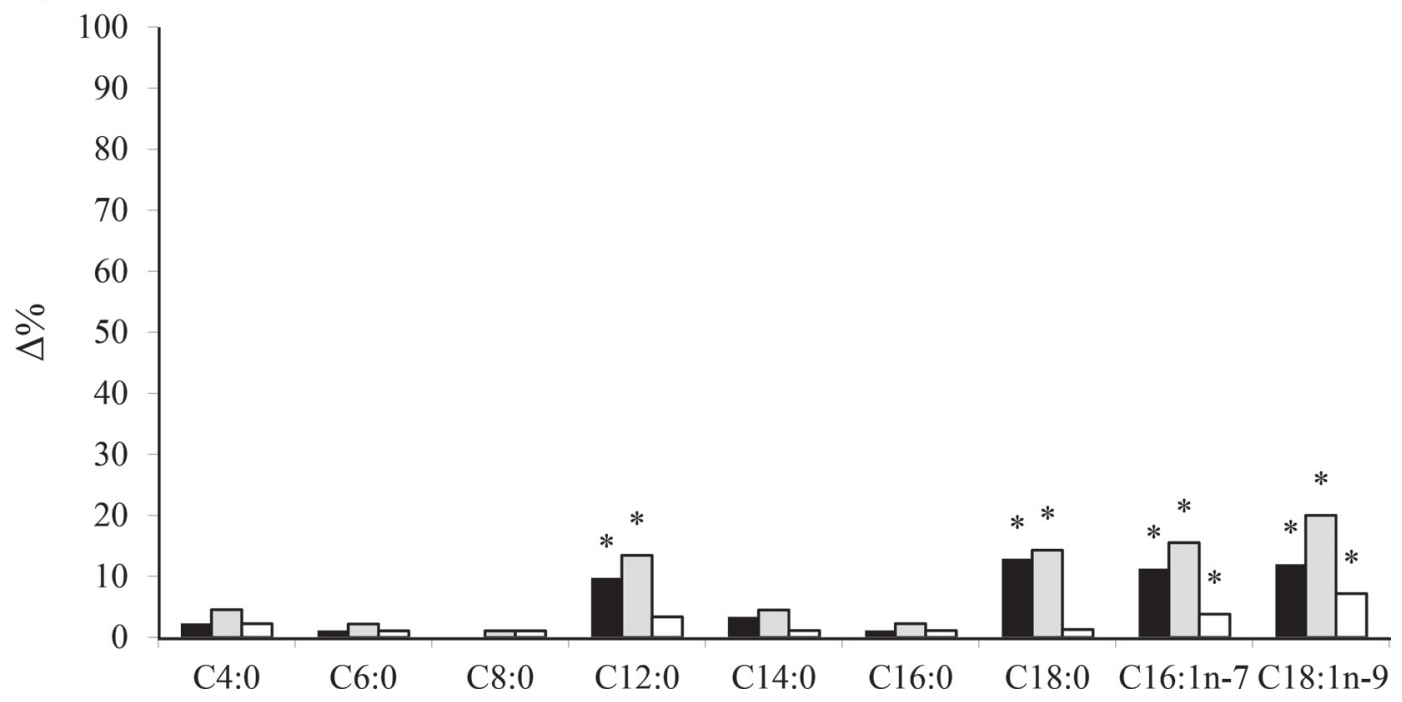

Figure 2. Percentage variation $(\Delta \%)$ of coefficient of determination in leave-one-out cross-validation $\left(\mathrm{R}_{\mathrm{CV}}^{2}\right)$ of prediction models for $(\mathrm{A})$ milk fatty acids (FA) groups and (B) individual milk FA (expressed in $\mathrm{g} / 100 \mathrm{~mL}$ of milk) obtained by comparing uninformative variable elimination (UVE) + partial least squares (PLS) regression versus PLS regression, genetic algorithm (GA) + PLS regression versus PLS regression, and GA + PLS regression versus UVE + PLS regression. The asterisk $\left(^{*}\right)$ above the bars indicates that percentage variations of coefficient of determination in leave-one-out cross-validation were significantly different at $P<0.05$.

comparisons of our results with those of the scientific literature difficult.

The practical utility of prediction models was ascertained using the RPD. When UVE + PLS regression was applied on traits expressed as grams per $100 \mathrm{~g}$ of total identified FA, only 1 FA group (SFA) and 2 individual FA (C6:0 and C12:0) exhibited RPD values equal or greater than 2, suggesting that prediction models for these features are useful for analytical purposes (Sinnaeve et al., 1994). In the case of GA + PLS regression, the practical utility of prediction models was reached also by MUFA, C4:0, C8:0, and C14:0. When the 2 statistical algorithms were applied to traits expressed on a milk basis, RPD values were much higher than those calculated for FA expressed as grams per $100 \mathrm{~g}$ of total identified FA and were always equal or greater than 2, with the only exception of PUFA for UVE + PLS.

A concern that could arise from the present study is that results are a function of the small data set and the lack of an external validation; therefore, an extra 
analysis was performed. The original data were divided in a calibration ( $60 \%$ of samples) and a validation set ( $40 \%$ of samples), selecting each sample at random into each data set. The calibration set was used to build MIRS prediction models for FA composition of milk both expressed on total identified FA and on a milk basis, and the validation set was used to validate the models. For each FA and within each statistical approach (PLS, UVE + PLS, and GA + PLS), this process was repeated 1,000 times. Despite the low number of samples included in the validation set, fitting statistics were stable across the 1,000 analyses (results not shown) and they were very similar to those calculated using the leave-one-out cross-validation.

\section{Computational Time}

All the analyses were performed using a quad-core $(2.40 \mathrm{GHz})$ laptop with $8 \mathrm{~Gb}$ of RAM and a 64-bit operating system. The UVE + PLS procedure required approximately $8 \mathrm{~min}$ to complete the analyses, whereas GA + PLS required an average of more than $1 \mathrm{~h}$ per trait. As reported by Ferrand et al. (2011), this limit can be justified if counterbalanced by a significant improvement of the accuracy. In the present study, the use of variable selection procedures before PLS regression improved $\mathrm{R}_{\mathrm{CV}}^{2}$ of prediction models for milk FA composition; thus, both UVE and GA are advisable to build more accurate equations compared with PLS regression only. Also, the improvement concerned mainly FA that are usually difficult to predict (e.g., PUFA). The comparison between UVE + PLS and GA + PLS showed that the second led to overall better accuracies than the first approach, especially when FA were expressed on a milk basis; thus, GA + PLS could be preferred in routine conditions. However, further research is needed to better compare the 2 variable selection procedures.

One feature that should be emphasized is the objectivity of the 2 selection algorithms. The construction of the UVE selection filter is absolutely not influenced by any external decision (Centner et al., 1996; Mehmood et al., 2012) as well as the choice of the various parameters in the GA procedure (Leardi and González, 1998; Leardi, 2003; Ferrand et al., 2011).

\section{CONCLUSIONS}

Interest is growing in the determination of the fine composition of milk, and MIRS might be a fast and cheap technique to collect new phenotypes at both herd bulk and individual cow level. However, the routine application of prediction models requires the equations to be accurate. The present study highlighted that vari- able selection approaches (UVE and GA) before PLS analysis are useful to improve the accuracy of prediction for FA composition of milk. This would facilitate the use of prediction equations in the dairy industry for genetic purposes and for payment system. Further research will investigate the effect of sample size on fitting statistics of prediction models for milk FA composition. Also, a more comprehensive comparison between cross-validation and external validation is needed to quantify their effect on prediction models performance after different variable-selection approaches.

\section{ACKNOWLEDGMENTS}

The authors thank the milk laboratory of the South Tirol Dairy Association (Bolzano, Italy) for providing spectra data used in this study and the Breeders Association of Veneto region (Padova, Italy) for collaborating to sample collection. Technical support by Giulio Visentin, Giovanni Niero, and Sofia Ton (University of Padova) and the generosity of dairy farms who participated in the trial are also gratefully acknowledged.

\section{REFERENCES}

Centner, V., D. L. Massart, O. E. de Noord, S. de Jong, B. M. Vandeginste, and C. Sterna. 1996. Elimination of uninformative variables for multivariate calibration. Anal. Chem. 68:3851-3858.

Chun, H., and S. Keleş. 2010. Sparse partial least squares for simultaneous dimension reduction and variable selection. J. R. Stat. Soc. B Stat. Methodol. 72:3-25.

Coates, J. 2000. Interpretation of infrared spectra, a practical approach. Pages 10815-10837 in Encyclopedia of Analytical Chemistry, R.A. Meyers, ed. John Wiley \& Sons Ltd., Chichester, UK.

Comino, L., F. Righi, M. Coppa, A. Quarantelli, E. Tabacco, and G. Borreani. 2015. Relationships among early lactation milk fat depression, cattle productivity and fatty acid composition on intensive dairy farms in northern Italy. Ital. J. Anim. Sci. 14:350-361.

De Marchi, M., M. Penasa, A. Cecchinato, M. Mele, P. Secchiari, and G. Bittante. 2011. Effectiveness of mid-infrared spectroscopy to predict fatty acid composition of Brown Swiss bovine milk. Animal 5:1653-1658.

De Marchi, M., V. Toffanin, M. Cassandro, and M. Penasa. 2014. Invited review: Mid-infrared spectroscopy as phenotyping tool for milk traits. J. Dairy Sci. 97:1171-1186.

Faber, N. M., M. J. Meinders, P. Geladi, M. Sjöström, L. M. C. Buydens, and G. Kateman. 1995. Random error bias in principal component analysis. Part I. Derivation of theoretical predictions. Anal. Chim. Acta 304:257-271.

Ferrand, M., B. Huquet, S. Barbey, F. Barillet, F. Faucon, H. Larroque, O. Leray, J. M. Trommenschlager, and M. Brochard. 2011. Determination of fatty acid profile in cow's milk using mid-infrared spectrometry: Interest of applying a variable selection by genetic algorithms before a PLS regression. Chemom. Intell. Lab. Syst. 106:183-189.

Geladi, P., and B. R. Kowalski. 1986. Partial least-squares regression: A tutorial. Anal. Chim. Acta 185:1-17.

Gottardo, P., M. De Marchi, M. Cassandro, and M. Penasa. 2015. Technical note: Improving the accuracy of mid-infrared prediction models by selecting the most informative wavelengths. J. Dairy Sci. 98:4168-4173.

Haaland, D. M., and E. V. Thomas. 1988. Partial least-squares methods for spectral analyses. 1. Relation to other quantitative calibra- 
tion methods and the extraction of qualitative information. Anal. Chem. 60:1193-1202.

Helland, I. S. 2001. Some theoretical aspects of partial least squares regression. Chemom. Intell. Lab. Syst. 58:97-107.

Hewavitharana, A. K., and B. van Brakel. 1997. Fourier transform infrared spectrometric method for the rapid determination of casein in raw milk. Analyst (Lond.) 122:701-704.

Leardi, R. 2003. Genetic algorithm-PLS as a tool for wavelength selection in spectral data sets. Data Handl. Sci. Technol. 23:169-196. http://dx.doi.org/10.1016/S0922-3487(03)23006-9.

Leardi, R., and A. L. González. 1998. Genetic algorithms applied to feature selection in PLS regression: how and when to use them. Chemom. Intell. Lab. Syst. 41:195-207.

Lopez-Villalobos, N., R. J. Spelman, J. Melis, S. R. Davis, S. D. Berry, K. Lehnert, S. E. Holroyd, A. K. H. MacGibbon, and R. G. Snell. 2014. Estimation of genetic and crossbreeding parameters of fatty acid concentrations in milk fat predicted by mid-infrared spectroscopy in New Zealand dairy cattle. J. Dairy Res. 81:340-349.

Martens, H., and T. Naes. 1989. Multivariate Calibration. J. Wiley and Sons, Chichester, UK.

McDermott, A., G. Visentin, M. De Marchi, D. P. Berry, M. A. Fenelon, P. M. O'Connor, O. A. Kenny, and S. McParland. 2016a. Prediction of individual milk proteins including free amino acids in bovine milk using mid-infrared spectroscopy and their correlations with milk processing characteristics. J. Dairy Sci. 99:3171-3182.

McDermott, A., G. Visentin, S. McParland, D. P. Berry, M. A. Fenelon, and M. De Marchi. 2016b. Effectiveness of mid-infrared spectroscopy to predict the color of bovine milk and the relationship between milk color and traditional milk quality traits. J. Dairy Sci. 99:3267-3273.

Mehmood, T., K. H. Liland, L. Snipen, and S. Sæbø. 2012. A review of variable selection methods in partial least squares regression. Chemom. Intell. Lab. Syst. 118:62-69.
Niero, G., M. Penasa, P. Gottardo, M. Cassandro, and M. De Marchi. 2016. Short communication: Selecting the most informative mid-infrared spectra wavenumbers to improve the accuracy of prediction models for detailed milk protein content. J. Dairy Sci. 99:1853-1858

Palmquist, D. L., and T. C. Jenkins. 2003. Challenges with fats and fatty acid methods. J. Anim. Sci. 81:3250-3254.

R Core Team. 2015. R: A language and environment for statistical computing. R Foundation for Statistical Computing, Vienna, Austria. Accessed Nov. 15, 2015. https://www.r-project.org/.

Sinnaeve, G., P. Dardenne, R. Agneessens, and R. Bistom. 1994. The use of near infrared spectroscopy for the analysis of fresh grass silage. J. Near Infrared Spectrosc. 2:79-84.

Soyeurt, H., P. Dardenne, F. Dehareng, G. Lognay, D. Veselko, M. Marlier, C. Bertozzi, P. Mayeres, and N. Gengler. 2006. Estimating fatty acid content in cow milk using mid-infrared spectrometry. J. Dairy Sci. 89:3690-3695.

Soyeurt, H., F. Dehareng, N. Gengler, S. McParland, E. Wall, D. P. Berry, M. Coffey, and P. Dardenne. 2011. Mid-infrared prediction of bovine milk fatty acids across multiple breeds, production systems, and countries. J. Dairy Sci. 94:1657-1667.

Thomas, E. V., and D. M. Haaland. 1990. Comparison of multivariate calibration methods for quantitative spectral analysis. Anal. Chem. 62:1091-1099.

Visentin, G., A. McDermott, S. McParland, D. P. Berry, O. A. Kenny, A. Brodkorb, M. A. Fenelon, and M. De Marchi. 2015. Prediction of bovine milk technological traits from mid-infrared spectroscopy analysis in dairy cows. J. Dairy Sci. 98:6620-6629.

Wehrens, R. 2011. Chemometrics with R-Multivariate Data Analysis in the Natural Sciences and Life Sciences. Springer, Heidelberg, Germany. 